\title{
Use Strategic Assessment to Demonstrate Impact: a case study at the HKUST Learning Commons
}

Assessment has become a core part of library culture in developing and evaluating new services, space and facilities. As more libraries worldwide embrace the service model of "commons", librarians have been exploring effective means to assess this new genre of learning space and support.

"Information commons can enhance learning, provide an environment for students that is both academic and social, and foster a sense of community on campus" (Lippincott, 2006). Although she uses the term "information commons", Lippincott's comment is valid for a wide range of new learning space that modern libraries develop. The conversion of traditional library space to the "commons" model has also been a trend in Hong Kong higher education in the past decade. The migration was accelerated by the university program reform in Hong Kong: all government-funded higher institutions in the city changed from a 3-year program structure to a 4-year one in Fall 2012. In this connection, many university libraries embarked on capital projects to create commons-style space to receive the new learning landscape. The Hong Kong University of Science and Technology Library (HKUST Library) completed the Learning Commons (LC) in Spring 2012. With the goal of being a supportive and engaging learning space, the LC provides 600 seats in the 1,800 square meters floor space, which are configured as a variety of styles and purposes: individual or collaborative, relaxed or focused, open or enclosed, flexible or designated, traditional or multimedia, high-touch or high-tech. The LC is an integral part of the Lee Shau Kee Library Building, yet it is capable to open $24 \times 7$ independently; this became one of its key feature.

Usage statistics, taken regularly, indicated a high traffic volume at the LC. However, the Library lacked qualitative data about users' experience. A strategic assessment was hence conducted in Spring 2013 to further evaluate users' perception and to identify areas for improvement. This article describes the assessment exercise, from planning, implementing, to data processing and presentation. Carried out strategically, the assessment did more than giving us a list of actions for service and facility improvement; it provided strong evidence for the Library to demonstration its impact on students' life, and brought us recognition and support from the university administration. The outcome was beyond the Library's original expectation.

\section{Background for Assessment at HKUST LC}

"If we want to engage students in library spaces, it is imperative we discard traditional views of library space and furniture" (Webb, et al., 2008). Libraries and librarians who develop commons understand this well all along, but the same cannot be assumed for other stakeholders in a university community. Therefore, when the LC was opened in February 2012, we, the HKUST Library, carried out a proactive promotion program. One of the key messages we conveyed in the promotion was that the LC was not 
just another quiet study space in the campus (Chan \& Wong, 2013). Although that initial promotion was a success, we felt that we could not stay content with that. The need to identify evidence to demonstrate the value of the $\mathrm{LC}$ remains strong.

After the LC opened in February 2012, services and facilities were fine-tuned, developed and consolidated gradually in the subsequent months. During the first year of operation, users got familiar with the ways they could use the space, they learnt and got accustomed to the rules in the Learning Commons. Staff also got more comfortable and confident in the mode of service provision. Routines and workflows were refined and smoothed out; the pool of accessory items for loan at the service desk was enriched; the team of student workers were growing and getting strong. Supporting learning activities was one of the main goals of the LC. Some regular tutorial programs and career programs were established early on; increasing number of teaching and service units in the university followed suit to explore how they could use the space to enrich students' learning or provide services. In terms of operation hours, the LC tested opening 24 hours during the examination weeks in Fall 2012. It was ready to extend the long hours to most of the Spring Semester 2013. In short, by Spring 2013, the Learning Commons has grown into a vibrant learning space.

Traditional metrics have been collected from the start. Gate counts and head counts are taken multiple times daily. Computer logins, printer logs, software usage and circulation figures of accessory items for loan (such as HDMI cable, USB, whiteboard markers, etc.) at the service desk are compiled as monthly report. Question log at the service desk and room booking records can be retrieved from the corresponding databases as needed. All these figures showed either heavy usage or healthily growing trend in the first year of operation.

As the LC aims to be a supportive and engaging learning space, we value users' experience as much as, if not more than, traditional metrics of input and output. Regular usage monitor informs us our space and facilities are well-utilized, but how about students' perception? Do they see our space as engaging? Do they have positive experience with the LC? Do they like our space? Are we supporting their learning? Are we simply one of the many study areas in the campus? What can be added or changed at the LC?

In order to answer these questions, we carried out an assessment exercise in Spring 2013.

\section{Literature review}

Coordinated assessment program is crucial for a commons to reveal needed change, justify additional funding, and demonstrate its contribution to teaching and learning (Lippincott, 2006). How has the library world carried out assessment to achieve these important outcomes? The volume of library literature on this topic presents a variety of themes, including assessment objectives, methods, implementation, findings, impacts and lessons learnt.

In terms of methods of assessment, Lippincott listed measures such as counts (gate record, use of facilities, and questions asked), surveys (use, satisfaction and quality perception) and focus groups (Lippincott, 2007). These methods have been frequently used by libraries in all functional areas. There has also been a shift from using traditional measures of investment (inputs) and activity (outputs), to 
measuring library's influence on enhanced learning and quality research (outcomes) (Forrest \& Williamson, 2003). This trend is particularly salient for evaluating commons, where librarians need to explore more non-traditional measure in their assessment effort. Most assessment cases used multiple channels to collect data. For example, a multifaceted approach was used at University of Houston Learning Commons. Among the methods implemented there were paper survey, headcounts, feedback, questions tracking. As a result of having gathered information from the different forms of assessment, the UH Learning Commons successfully achieved its goal of creating and maintaining an undergraduate research support facility (McKee, 2010).

Some libraries focused on understanding users' space preferences and usage volume. Such data usually became the driving force for new facilities and improvement. At Indiana University, systematic observation of non-computer seating areas inside and outside the library revealed students' needs and choices for individual and group study. The findings helped the library to tailor its designs to fit into its own campus needs (Applegate, 2009). Using login data from library computers, Weessies analyzed and characterized space preferences of computer users in Michigan State University main library. She concluded, '[b]y paying close attention to student use of space, the academic library can - not so much repurpose itself - but bring new life to the user experience" (Weessies, 2011). At University of Huddersfield, a combination of qualitative and quantitative methods was selected to gather data on various levels, so as to gather a broad picture of student space selection as well as actual usage. Methods included staff observation and comments, subject desk enquires, weekly reflective logs, quantitative study of space usage, students' learning logs, comments and questionnaires (Ramsden, 2011). Whitchurch monitored group use over two years, proving that the commons has been heavily used as an area for group study (Whitchurch, 2009). Emory libraries studied data on group study use and occupancy data as the basis for decision-making about space furnishing and operation hours; while University of Missouri-Kansas City Libraries evaluated their information commons by building usage data and LibQUAL+. Both sites used continuous assessment to drive incremental and phased improvement (Forrest \& Bostick, 2013).

Apart from usage, librarians have been paying more and more attention to assessing impact. "The library's success is measured not by what it has or what it does, by how it's used or the activities it supports, but what happens as a result of those activities and encounters, the impacts or outcomes of what goes on in the library" (Forrest \& Bostick, 2013). The University of Dayton employed a variety of methods to understand how library space may have an impact on and contribute to student learning behaviors. The multi-method approach, comprised of surveys, Web poll, video study and data from National Survey of Student Engagement (NSSE), helped library to understand the full experience of students of the space. Their findings confirmed the type of learning environments that students prefer: spaces that are comfortable, spaces that facilitate interpersonal communication, spaces that can be controlled, and areas that promote the integration of basic human needs and desires, such as eating, drinking, and enjoyment, with learning activities (Webb, et al., 2008).

Looking at users' experience is one way of assessing impact. The idea of "Library as space" brings assessment focus to "user experience". As Forrest and Bostick pointed out, "[g]oods are useful and service is helpful, but experiences are memorable. Experiences leave a lasting impression and can be 
transformative" (Forrest \& Bostick, 2013). This echoes with the trend of moving from traditional usage measures to assessing library influence. Users' experience and perceptions have become an important pool for librarians to tap into while planning assessment. However well designed and powerful the environment is, however well implemented by staff, it is "students perceptions of that learning environment [that] will determine what kind of learning activities will be employed, and of what the learning outcomes will be" (Könings, et al., 2005).

\section{Assessment Strategy}

The assessment exercise at the Learning Commons of the HKUST Library, conducted in Spring 2013, aimed to help the Library understand students' and service partners' experience of using the LC, and to identify services and facilities that require improvement. The assessment was coordinated by the Learning Commons Manager. Planning and strategy was discussed in the Learning Commons Working Group, which comprised of 4 librarians. The implementation and logistics was a concerted effort of librarians, support staff, and student helpers at the HKUST Library.

The approach of the assessment was exploratory. Other than the aims stated above, namely, finding out users' experience and actionable improvement, we did not pre-define specific assumptions or research questions.

We implemented the assessment in three strategic phases:

1. Student focus groups

2. Student survey

\section{Service partner survey}

The instrument of each phase was strategically designed based on the findings of and insight from the previous one.

The findings, complementing the usage statistics taken regularly, would become the basis for the HKUST Library to fine-tune existing provision, develop new services and facilities, and explore new partnerships.

\section{Student Focus Groups}

How do libraries assess experience? For an open, flexible learning environment like a commons, what is the value that makes it a supportive and engaging space? It is difficult to determine "the intrinsic value of an environment for students. Socialization is very important, as are intellectual development and cultural awareness opportunities these places bring" (Dewey, 2007). In such context, focus group is an effective strategy that can help us explore unanticipated issues.

We conducted three focus groups in March 2013, one used English as the medium, two in Cantonese, the Chinese dialect spoken in Hong Kong. HKUST has about $25 \%$ non-local students, who come from countries all over the world, including those from Mainland China. The official medium of instruction at the University is English. However, most local Hong Kong students have strong preference to using their 
mother tongue rather than English to express themselves freely. As a result, 10 students who signed up for the English session were non-local, some were undergraduates and some postgraduates. The first Cantonese group attracted 8 undergraduate students, overwhelmingly local. The second Cantonese group was attended by 3 local first-year undergraduate students. Incidentally, the three groups represented different faces of our student body, revealed to us the diversity of student profiles and their varied needs.

The focus groups were moderated by librarians. The discussion was kept as open as possible, so that students could share freely their experience and perception of using the LC. To achieve this, the moderators were reminded to trigger as little guiding or priming as possible when initiating a discussion.

Overall, the three sessions were all very positive and lively. Very importantly, they helped us to shape two powerful questions for the student survey in the second phase of the assessment. We found that most interesting comments from students that could demonstrate the value of the space converged towards two themes: what the LC means to them in their campus life; and how the LC has changed their use of the Library. With this insight, we phrased two key questions in the subsequent student survey:

- In one sentence, describe what the Learning Commons means to you.

- How has the Learning Commons changed the way you use the Library?

\section{Student Survey}

Questionnaire survey is a powerful tool to provide quantitative data. An effective questionnaire should be brief and concise; all questions should lead to findings that are meaningful, usable or actionable. We brainstormed to craft and design questions, and to identify questions that could give us valuable information or actionable follow-up. We made an effort to deselect questions which would lead to answers that had very little impact in terms of follow up actions, despite the temptation that some of such answers were interesting to know from curious librarians' perspective.

The resulting questionnaire had 8 questions. Besides simple demographic questions and the two key questions that were inspired by the focus groups discussion, others asked students for their frequency of use, perceived importance of various features of the LC, suggestions and any negative experience ever encountered at the LC.

The questionnaire was available in both print form and online. Respondents could choose to enter a lucky draw for a HK\$50 supermarket coupon after they completed the questionnaire. The survey was conducted in the last two weeks of March 2013. We received an encouraging number of 886 responses.

\section{Service Partner Survey}

This last phase of the assessment was a satisfaction survey for the service partners of the LC. In relation to designing this survey, we employed the experience from designing the student survey. We invited faculty, teaching departments, and academic support units to share with us their experience of using the LC for teaching or program. The questionnaire was brief: it contained 6 questions for respondents who had conducted programs at the LC, and 4 questions for those who had not. The survey was opened for 
two weeks in April 2013. We received 32 completed questionnaires at the end of the period. Twenty nine respondents had experience offering programs at the LC, 3 had not.

\section{Data Processing and Findings}

The assessment produced a good amount of data, full of encouraging feedbacks in students' authentic voice. The quantitative data from the two surveys was tabulated. The qualitative data, including the focus group notes and open comments from the surveys, was manually coded and categorized.

\section{Focus Groups}

We analyzed the written notes taken in the three focus group discussions, categorized the comments into a few major themes: what students liked, what students disliked, what LC meant to their campus life, how they perceived the connection between LC and the Library, and suggestion for new services or facilities.

This exercise helped the Library to have a better glimpse into our students' world. Apart from the verbal comments, we also made observations of the tone and dynamics of the discussions. "Reading between the lines" gave us insights into students' situation and needs. For example, the English speaking session was the liveliest one among the three groups. This group of non-local students, including postgraduates, raised a lot of high quality feedbacks. They spent much of their time in the LC for study and social purposes. Being far from their homes and families, an integrated space like the LC became their best substitute for a space that provides a sense of belonging. The accentuation of cultural diversity at the LC will be a factor for the Library to consider when responding to students' behaviors or requests, and when planning new services and facilities. On the other hand, the second Cantonese session attended by three local freshmen had a completely different tone. These students were not as outspoken and confident as the English group; they did not use the Library and the LC as much either. However, they brought our attention to a service gap: the LC and the Library had not been strong enough in engaging local students in an authentic way. This revelation will have implications on the Library's future planning of learning programs and user outreach programs.

\section{Student Survey}

The survey received 886 completed questionnaires. This enthusiastic response went beyond our expectation. This is encouraging not only because a larger dataset would have stronger representation, but we also took it as a positive sign of students' sentiment towards the LC.

From the quantitative responses, we identified the three most-popular activities students used the LC for: "self-studying", "meeting friends", and "using computers". For the perception of importance, 90\% of the respondents rated the LC as a whole as "Very Important" or "Important"; followed by $87 \%$ rating 24-hour operation as "Very Important" or "Important". Regarding new services or facilities, $44 \%$ of the respondents suggested more technology workshops, and $41 \%$ suggested more cultural activities.

Analyzing the written comments was a more complex process. The amount of comments we received was large. For the two key questions, there were 569 replies to "Q5. In one sentence, describe what the 
Learning Commons means to you"; and 473 replies to "Q6. How has the Learning Commons changed the way you use the Library". The comments were browsed manually by the Learning Commons Manager to identify main themes. Each comment was then coded in an Excel sheet according to two aspects: the theme(s) and distinctiveness. For example, one comment responding to Question 5 was "it was a place for me to study overnight"; this descriptive comment was grouped under the theme "opening hours", and rated as low in "distinctiveness". While a more emotional comment "It is one of the reasons of my good GPA!" was rated as high "distinctiveness". The grouping and rating facilitated effective filtering for summarizing the findings. The theme code enabled us to count the comments in various areas and made inferences from them. The "distinctiveness" code let us easily retrieve feedbacks that were particularly informative or revealing, as well as identifying interesting quotes that reflect students' perception to the $L C$.

Over 1,700 written comments were collected from the survey; all were categorized and coded. Four themes were identified for each of Question 5 and 6. For Question 8, which asked students to share any unpleasant experience, over 20 themes were coded. More than 40 comments from Question 5 and 6 were coded as "very distinctive". For example, one student said, "I am proud of the LC, which I would regard as an indispensable part of the library." Another said, "I spend all my time in Learning commons for study when I have no class. My life!"

Authentic students' comments like these are particularly powerful. Overall, the rich pool of students' testimony told us a lot about how they enjoyed using the LC as a flexible study space and social space, and how the LC has positive impact on the usage and perception on the Library.

\section{Service Partner Survey}

This survey received 32 completed questionnaires: 29 had offered programs at the LC; 3 had not. The number of response rate was within our expectation. The $L C$ has been actively promoting its space for teaching faculty and academic support units to conduct learning or service programs. From the opening in Spring 2012 to the time of assessment in Spring 2013, the number of service partners had been growing all along. In Spring 2013, about 10 units offered more than a dozen of activities at the LC, some were regular daily or weekly arrangement (such as tutorials and counseling sessions), some were onetime events (such as workshops and talks). Therefore, we expected the volume of response to this partner survey to be in the range of dozens. We were also aware that, as the questionnaires were filled out by anonymous individuals, they did not correspond to individual partner units, nor individual programs or events.

Among those respondents who used the LC before, 58\% had classes or workshops, $27 \%$ operated career advising programs. Regarding their satisfaction level, $81 \%$ responded their programs went well; satisfaction rating on all aspects of Library support was on the positive side.

\section{Presenting Findings}

Finishing data analysis was not the end of the exercise. On the contrary, the assessment exercise came to the pivotal point at the stage of summarizing and presenting the findings. The success of this 
assessment depended on the critical step of drawing meaningful conclusions from such a rich and diverse dataset; and conveying them to the relevant parties in an effective ways.

No doubt, we were excited with the positive response. With the rich set of quantitative and qualitative data, we were confident that we achieved the assessment objectives of finding out users' experience and actionable improvements. At the same time, the encouraging response compelled us to share it with our university administrators. We realized that we needed to relay the findings to different groups of stakeholders for very different purposes.

\section{Library Action Report and Executive Report}

In light of the situation, we presented the findings in two separate reports, one for internal use by the Library, and one for submitting to university administrators.

The Library Action Report is a 17-page Word document. It was written for the Library's internal dissemination and discussion. It highlights the key findings, and includes a list of suggested follow-up actions to be deliberated further by various functional units in the Library. Supplementary files including a 50-slides PowerPoint of data charts, lists of written comments and other data files were made available in the Library intranet. The report was widely discussed in different working groups and committees within the Library in May 2013. As a result, a number of changes were made or planned in subsequent months. Among many others, these are a few of the actions we took to address students' feedback:

- The rules for study room booking were refined

- A new reminder about the rules was added at multiple points at the online room booking process

- Patrols were strengthened to maintain the quality of the study space

- Extra paper towel dispensers were added to washrooms

- Art work and plants were added to the space

Apart from these changes that could be made within a relatively short time, the findings continue to inform future library decisions on service and facilities in the long terms.

The Executive Report, on the other hand, was a succinct 10-page summary without appendix. The findings were summarized as three descriptive themes:

1. The LC supports student learning by functioning as their primary study space

2. The $L C$ is too small and overcrowded to meet the high student traffic

3. Service partners have positive experience of operating learning activities at the LC

Each theme was elaborated and supported by selective, relevant figures and evidence from the assessment data. Among others, highlighted data includes:

- $90 \%$ of students rated LC as "Important" or "Very important"

- Not a single service partner gave any unsatisfactory rating on the services and facilities 
- Many students love LC because it serves their multiple needs anytime, and pleasantly

To help our administrators visualize students' experience and perception of the LC, the written comments responding to "Q5. In one sentence, describe what the Learning Commons mean to you" were consolidated and presented as a tag cloud (Figure 1) using the free web software at tagul.com.

The crisp and visual presentation successfully conveyed the powerfully positive tone of the assessment result to our university administrators, who were impressed by and convinced of the impact of the LC as an engaging learning space. It strengthened their trust to the Library in operating learning space with success, and helped garner their support for Library's initiatives on developing more engaging space that students like and need. As the assessment feedbacks strongly pointed to a need to expand the capacity of the Learning Commons, the University Librarian at HKUST took the initiative to explore options for expansion. Her intention received solid support from university administration.

\section{Summary}

This is a story about how strategic assessment can highlight library's impact and bring real changes to library services and facilities.

The Learning Commons at HKUST Library aims to be an engaging and supportive space for student learning. It was the first of its kind in the HKUST campus. After the first year of operation, during which services and facilities were consolidated in steps, the Library conducted this assessment to find out users' experience, review what have been done right for our users, and what could be changed for the better.

The three-phased assessment was strategically planned and implemented in Spring Semester 2013. Each phase was built on the previous one so that we could make the instrument as effective as possible to achieve the assessment goal. The response was very encouraging, especially the student survey which received 886 completed questionnaires full of students' authentic comments. We charted quantitative data and coded qualitative data in meaningful ways. That brought the effort to its pivotal point: how to present the findings effectively in order to bring changes?

We faced the challenge by making two separate reports that target the two very different audience for difference impact: librarians for actionable follow-up, and university administrators for demonstrating value.

As a result of the process, HKUST Library refreshed our understanding of students' need. We identified a list of facility and service improvement for both student users and service partners. An unexpected outcome was the university trust and support we gained after we used a concise and visual report to demonstrate the value of our space to students' learning. The trust will result as physical change when the Library develops more space and facilities that continue to support students in such an engaging way. However, we do not stop here. "Evaluation must be an iterative process in order to effectively and continually measure learner behaviors and pedagogic changes". Academic libraries with foresight 
respond to and drive the strategic change that influence teaching and learning within their institutions (Appleton, et al., 2011). HKUST Library strives to remain on that par.

\section{Bibliography}

Applegate, R., 2009. The library is for studying: student preferences for study space. The Journal of Academic Librarianship, 35(4), pp. 341-346.

Appleton, L., Stevenson, V. \& Boden, D., 2011. Developing learning landscapes: academic libraries driving organisational change. Reference Services Review, 39(3), pp. 343-361.

Chan, D. \& Wong, G., 2013. If you build it, they will come: An intra-institutional user engagement process in the Learning Commons. New Library World, 114(1/2), pp. 44-53.

Dewey, B. I., 2007. Linking the Information Commons to Learning: how to measure success. Vintage Point Series, pp. 6-12.

Forrest, C. \& Bostick, S. L., 2013. Welcoming, flexible, and state-of-the-art: approaches to continous facilities improvement. IFLA Journal, 39(2), pp. 140-150.

Forrest, C. \& Williamson, A., 2003. From inputs to outcomes: measuring library service effectiveness through user surveys. Georgia Library Quarterly, 40(2), pp. 12-18.

Könings, K. D., Brand-Gruwel, S. \& van Merriënboer, J. J. G., 2005. Towards more powerful learning environments through combining the perspectives of designers, teachers, and students. British Journal of Educational Psychology, 75(4), pp. 645-660.

Lippincott, J. K., 2006. Linking the information commons to learning. In: D. G. Oblinger, ed. Learning Spaces. s.I.:EDUCAUSE.

Lippincott, J. K., 2007. Assessing information commons. Vantage Point Series, pp. 2-5.

McKee, N. P., 2010. A multifacted approach to the assessment and evaluation of learning commons services and resources. College \& Undergraduate Libraries, 17(2-3), pp. 297-309.

Ramsden, B., 2011. Evaluating the impact of learning space. Reference Services Review, 39(3), pp. 451464.

Webb, K. M., Schaller, M. A. \& Hunley, S. A., 2008. Measuring library space use and preferences: charting a path toward increased engagement. portal: Libraries and the Academy, 8(4), pp. 407-442.

Weessies, K. W., 2011. A locational analysis of academic library computer use. Reference Services Review, 39(3), pp. 465-481.

Whitchurch, M. J., 2009. Evaluating group use of the information commons. College \& Undergraduate Libraries, 16(1), pp. 71-82. 
